\title{
On the evolution of simulated galaxies: the mass dependence in metallicity gradients
}

\author{
Maider Miranda ${ }^{1,2}$, Patricia Sánchez-Blázquez ${ }^{2}$, \\ Chris B. Brook ${ }^{2}$ and Brad K. Gibson ${ }^{3,1}$ \\ ${ }^{1}$ University of Central Lancashire, Preston, UK \\ email: msancho@uclan.ac.uk \\ ${ }^{2}$ Universidad Autónoma de Madrid, Madrid, Spain \\ ${ }^{3}$ University of Hull, Hull, UK
}

Making use of a fiducial set of simulated disc galaxies spanning a wide range of mass, we examine the influence of stellar mass in the radial metallicity gradients and compare to observational trends from Ho et al. (2015).

The simulations employed here are drawn from the MaGICC project (Brook et al. 2012; Obreja et al. 2014). They form a homogeneous and complete suite of field galaxies covering a wide mass range, with Milky Way analogues, irregulars and dwarfs.

We study gas-phase radial oxygen gradients overplotting observations from Ho et al. (2015) and simulations (Fig. 1). Observations suggest that the mean gradient steepens when moving to lower galactic masses. MaGICC galaxies do not reproduce the measured metallicity distribution. Our gradients are too flat for stellar masses $M_{\star}<10^{9} M_{\odot}$, and this behaviour maintains at different redshifts. The total metal content is consistent with empirical scaling relations (Pilkington et al. 2012), but distributed incorrectly. We propose a mass-dependent modulation of feedback and/or star formation efficiency. Implementing such a mass-dependency must be handled with care, in order not to violate said empirical scaling relations.

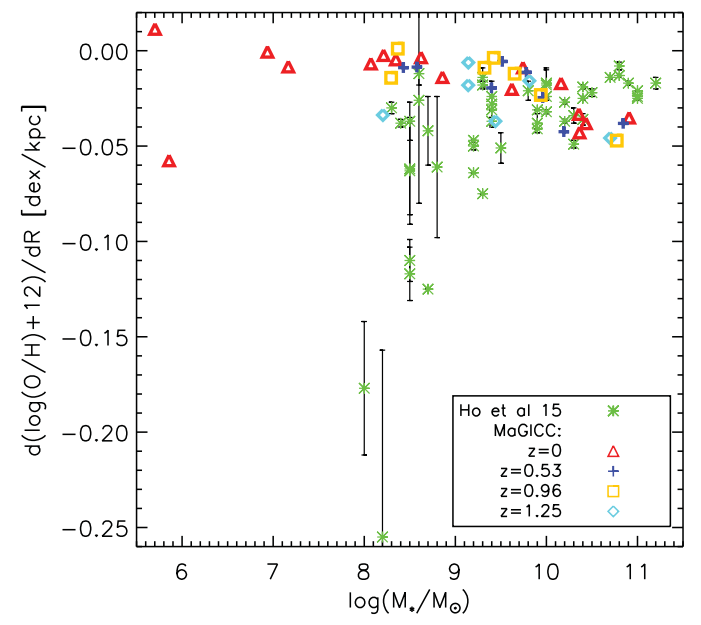

Figure 1. Oxygen gradient versus stellar mass, including observations from Ho et al. (2015) (green asterisks) and MaGICC simulations at different redshifts $(\mathrm{z}=0$ in red triangles, $\mathrm{z}=0.53$ in blue crosses, $\mathrm{z}=0.96$ in orange squares and $\mathrm{z}=1.25$ in cyan diamonds). Each symbol indicates an individual galaxy.

\section{References}

Brook, C. B., Stinson, G., Gibson, B. K., Wadsley, J., \& Quinn, T. 2012, MNRAS, 424, 1275

Ho, I.-T., Kudritzki, R.-P., Kewley, L. J., Zahid, H. J., et al. 2015, MNRAS, 448, 2030

Obreja, A., Brook, C. B., Stinson, G., Domínguez-Tenreiro, R., et al. 2014, MNRAS, 442, 1794

Pilkington, K., Gibson, B. K., Brook, C. B., Calura, F., et al. 2012, MNRAS, 425, 969 